\title{
Inhaltsverzeichnifs des neun und vierzigsten Bandes, nach den Gegenständen.
}

\section{Reine Mathematik.}

Nr. der bhandlung.

1. A n a l y s i s.

6. Beiträge zur Lehre von den Kettenbrüchen; nebst einigen Anwendungen $)^{\text {Heft. Seite. }}$ I. 66 auf die Berechnung der Wurzeln von Gleichungen. Von Hrn. Dr. Ottinger, ord. Prof. der Mathematik an der Universität zu Freiburg im Breisgau. .)

8. Rectification des formules qui expriment le nombre des décompositions d'un nombre donné en deux carrés. Par M. Paul Volpicelli, professeur à l'université de Rome.

10. Mémoire sur la résolution de l'équation indéterminée $a x+b k y=z\left(x^{2}+k y^{2}\right)$. Par Mr. Oltramare, prof. de math. supér. à l'académie des sciences de Genève. . . . . . . . . . . . . . . . . . . . . . . .

11. Note sur les relations qui existent entre les formes quadratiques des nombres premiers. Par le même. . . . . . . . . . . . . . . . II. 151

12. Mémoire sur la détermination des racines primitives des nombres premiers. Par le même. . . . . . . . . . . . . . . . . . . . . . II. 161

13. Recherches sur la résolution des équations de tous les degrés. Par M. le colonel $\boldsymbol{F}$. Theremin à Saratoff en Russie. . . . . . . . . . . . III. 187

14. Ober Determinanten und ihre Anwendung in der Geometrie, insbesondere auf Curven vierter Ordnung. Von Herrn Otto Hesse, Professor an der Universität zu Königsberg. . . . . . . . . . . . . . . . . . I I

\section{Ge o metrie.}

1. Allgemeiner Satz über die lineale Erzeugung aller algebraischen Oberflächen. Von dem Herrn Prof. H. Gra/smann, Oberlehrer am Gymnasio zu Stettin.

2. Grundsätze der stereometrischen Multiplication. Von Demselben. . . .

3. Über die verschiedenen Arten dèr linealen Erzeugung algebraischer Oberflächen. Von Demselben. . . . . . . . . . . . . . . . . . 1.21

4. Die stereometrische Gleichung zweiten Grades, und die dadurch dargestellTen Oberflächen. Von Demselben. . . . . . . . . . . . . . .

5. Die stereometrischen Gleichungen dritten Grades, und die dadurch erzeugten Oberflächen. Von Demselben. . . . . . . . . . . . . . .

. Sur les différents genres de multiplication. Par M. Gra/smann, prof. des mathém. au collège de Steltin. 
Iv Inhaltsverzcichnifs des neun und vierzigsten Bandes.

Nr. der
Abhandlung.
14. Uber

14. Über Determinanten und ihre Anwendung in der Geometrie, insbesondere Heft. Seite. auf Curven vierter Ordnung. Von Herrn Otto Hesse, Professor an der Universität zu Kõnigsberg. . . . . . . . . . . . . . . . . . III. 243

15. Eigenschaften der Curven vierten Grads rūcksichtlich ihrer Doppeltangenten. Von Herrn Prof. J. Steiner zu Berlin. . . . . . . . . . . . . III. 265

16. Aufgaben und Lehrsätze. Von Demselben. . . . . . . . . . . . III. 273

17. Über die Doppeltangenten der Curven vierter Ordnung. Von Herrn Otto Hesse, Professor an der Universität zu Königsberg. . . . . . . . . IV. 279

18. Über algebraische Curven und Flächen. Vom Herrn Prof. J. Steiner zu Berlin.

3. M e c h a n i k.

20. Zur Theorie des Potentials. Von Herrn Jul. Weingarten, Stud. Math. zu Berlin. .. . . . . . . . . . . . . . . . . . . . IV. 367

II. Angewand te $\mathrm{M}$ a thematik.

19. Über den Unterschied zwischen theoretischen und practischen Zinsrechnungen. (Nachtrag zu der Abhandlung über Sparcassen im 39. Bande dieses Journals S. 183 etc.) Vom Herausgeber. . . . . . . . . . . . . IV. 349

Druckfehlerverzeichnifs zum 47ten, 48 ten und 49 ten Bande. . . . . . $\left\{\begin{array}{rr}\text { I. } 94 \\ \text { IV. } 370\end{array}\right.$ 